\title{
Pentacene Organic Thin Film Transistors with Anodized Gate Dielectric
}

\author{
Silke Goettling ${ }^{a}$, Jochen Brill ${ }^{a}$, Norbert Fruehauf ${ }^{a}$, Jens Pflaum ${ }^{b}$, Eduardo Margallo-Balbás ${ }^{c}$ \\ ${ }^{a}$ Chair of Display Technology, University of Stuttgart, Allmandring 3b, 70569 Stuttgart, \\ Germany; \\ ${ }^{b} 3^{r d}$ Institute of Physics, University of Stuttgart, Pfaffenwaldring 57, 70550 Stuttgart, \\ Germany; \\ ${ }^{c}$ Current address: Electronic Instrumentation Laboratory, Delft University of Technology, \\ Mekelweg 4, 2628 CD Delft, The Netherlands
}

\begin{abstract}
A low temperature high quality gate dielectric process for bottom gate organic thin film transistors (OTFT) is introduced which is compatible to plastic substrates. The $\mathrm{Al}_{2} \mathrm{O}_{3}$ dielectric is grown from the aluminum gate electrode by anodic oxidation at room temperature and exhibits an exceptionally good electrical performance even for thin layers of $50 \mathrm{~nm}$. Finding an electrolyte which significantly reduces dielectric charges was instrumental for the desired OTFT application. The electrolyte and substrate dependent behaviour was characterized and compared to different dielectrics to point out the advantages of anodic oxidized aluminum. The characteristics of pentacene bottom contact OTFTs realized with anodized $\mathrm{Al}_{2} \mathrm{O}_{3}$ gate dielectric on glass and plastic substrates are presented.
\end{abstract}

Keywords: Organic Thin Film Transistors, Pentacene, Gate Dielectric, Anodisation

\section{INTRODUCTION}

Thin film transistors (TFT) with organic semiconducting material instead of silicon as active layer have a big potential for flexible display technologies. These so called organic thin film transistors (OTFT) can be used in a wide range of applications where flexible materials and low cost are required. In contrast to silicon, the thermal, structural and mechanical properties of organic semiconductors are similar to those of flexible plastic substrates. Additionally, organic semiconductors are deposited with low temperature processes. Since the maximal thermal load for many low cost substrates is less than $200^{\circ} \mathrm{C}$, such low temperature processes are essential. One disadvantage of OTFTs is the low mobility resulting from weak Van der Waal bonds. These Van der Waal bonds dominate in organic semiconductors in contrast to the covalent bonds dominating in conventional semiconductors.

Developing a process for OTFTs on flexible plastic substrates requires low temperature processes for all layers. For conventional display applications $\mathrm{SiO}_{2}$ and $\mathrm{Si}_{3} \mathrm{~N}_{4}$ are used as dielectric layer. Since these insulating materials are deposited at around $280^{\circ} \mathrm{C}$ they have to be replaced by low temperature alternatives. Finding a low temperature dielectric with good electrical properties is one of the most important steps in developing OTFTs.

One basic requirement for a switching TFT in an active matrix addressed display is a high on-current $I_{\mathrm{ON}}$ and a low off-current $I_{\mathrm{OFF}}$. A high $I_{\mathrm{ON}}$ is required to allow fast switching of the pixel and a low $I_{\mathrm{OFF}}$ to avoid discharging of the pixel in its OFF-state. For a low leakage current through the gate dielectric, a high quality insulating layer with a low defect density must be used.

The influence of the mobility $\mu$, the gate capacity $C_{\mathrm{GS}}$, the width $w$ and length $l$ of the transistor channel, the voltage between gate and source $V_{\mathrm{GS}}$ and threshold voltge $V_{\mathrm{th}}$ on $I_{\mathrm{ON}}$ is given by ${ }^{1}$

$$
I_{\mathrm{ON}}=\mu C_{\mathrm{GS}} \frac{w}{2 l}\left(V_{\mathrm{GS}}-V_{\mathrm{th}}\right)^{2} .
$$

Further author information: (Send correspondence to Silke Goettling)

Silke Goettling: E-mail: lfb@lfb.uni-stuttgart.de, Telephone: +49 (0)711 6856904

Organic Field-Effect Transistors IV, edited by Zhenan Bao, David J. Gundlach, Proc. of SPIE

Vol. 5940 (SPIE, Bellingham, WA, 2005) · 0277-786X/05/\$15 · doi: 10.1117/12.613636 
The mobility $\mu$ and thus the $I_{\mathrm{ON}}$ is limited by the weak van der Waal bonds. It is possible to decrease the operating voltage $V_{\mathrm{GS}}$ and the threshold voltage $V_{\mathrm{th}}$ by using a dielectric with a high specific gate capacitance $C_{\mathrm{GS}}$. From

$$
C_{G S}=\frac{\varepsilon_{0} \varepsilon_{r}}{d}
$$

with $\varepsilon_{0}=8.85 \cdot 10^{-12} \mathrm{As} / \mathrm{Vm}$ can be seen that $C_{\mathrm{GS}}$, can be increased by selecting a dielectric with a high dielectric constant $\varepsilon_{r}$ and a low thickness $d$. To achieve dielectric layers with a high $C_{\mathrm{GS}}$ different attempts can be found in literature. ${ }^{2-4}$ The properties of oxides which are grown from Aluminum or Tantalum by anodic oxidation are suitable as dielectric layers in OTFTs since thin layers of about $50 \mathrm{~nm}$ can be reliably produced having $\varepsilon_{r}=9$ and $\varepsilon_{r}=27$ respectively. Anodic oxidation is a low temperature process which is important for the use with plastic substrates. Additionally, our experiments showed that the surface roughness decreased during the process. The surface of the anodized metals is smoothed and the defect density is reduced. A low surface roughness is important for the growth of evaporated organic semiconductors. ${ }^{5}$

\section{EXPERIMENTS AND RESULTS}

Different dielectric materials are investigated for their compatibility in organic thin film transistors. Two different organic and four inorganic materials are investigated. The organic dielectrics, a polyacryl and a polyimide, are deposited by spin coating. The inorganic materials are plasma enhanced chemical vapour deposited (PECVD) $\mathrm{Si}_{3} \mathrm{~N}_{4}$ and $\mathrm{SiO}_{2}$ layers which have been developed for polysilicon TFTs ${ }^{6}$ and anodically oxidized $\mathrm{Ta}_{2} \mathrm{O}_{5}$ and $\mathrm{Al}_{2} \mathrm{O}_{3}$.

In this work the transfer of OTFT processes to plastic substrates is important. Therefore a low temperature dielectric with good insulating properties is needed. Spin coating and anodic oxidation are low temperature processes. The deposition temperature for the PECVD $\mathrm{SiO}_{2}$ and $\mathrm{Si}_{3} \mathrm{~N}_{4}$ layers is $280^{\circ} \mathrm{C}$ and is not suitable for the application on plastic substrates. But they are useful to compare the proved dielectric properties with those of the low temperature ones. Since the anodized oxides fullfill the above mentioned requirements the anodization process is discussed in more detail.

\subsection{Anodisation process}

Figure 1 shows schematically the anodization process.

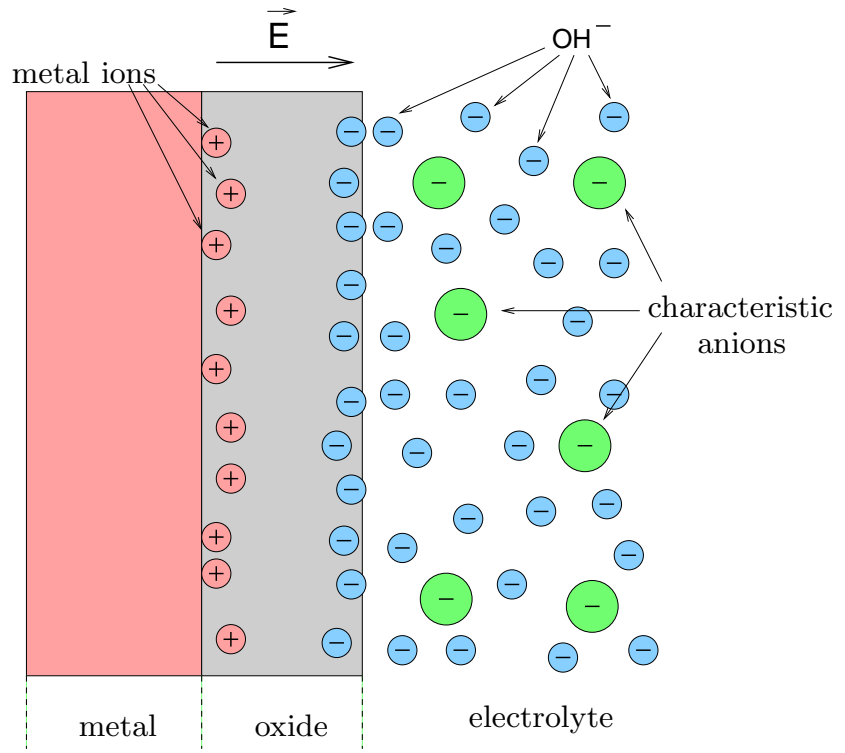

Figure 1. Model of the anodisation process. 
The substrate with a structured metal layer of aluminum or tantalum is used as the anode and a metal plate as the cathode in an electrolyte bath. The electrolyte must be electrically conductive and for the aluminum layer the $\mathrm{pH}$-value must be between 6 and 8 otherwise the aluminim layer is dissolved. During anodisation the current density is kept constant while the voltage increases linearly with the oxide thickness. Metal ions migrate onto the surface of the metal and react with $\mathrm{OH}^{-}$anions of the electrolyte. One part of the oxide grows into the metal and the other grows on the metal. The voltage defines the thickness of the oxide. When the desired tickness is reached, the voltage is kept constant so that the current density drops exponentially. ${ }^{7}$ Ethylenglycol and $\mathrm{H}_{2} \mathrm{O}_{2}$ buffered with ammoniumhydroxid to obtain a $\mathrm{pH}$-value of 7 are used as electrolyte to form $\mathrm{Al}_{2} \mathrm{O}_{3}$. Citric acid and $\mathrm{H}_{2} \mathrm{O}_{2}$ are used to grow $\mathrm{Ta}_{2} \mathrm{O}_{5}$. A varity of different possible electrolytes can be found in literature. ${ }^{4,7,8}$

\subsection{Characterisation of the dielectric}

Capacitors are first built on glass substrates to characterize and compare the different materials. The current density is measured while increasing the electrical field strength linearly by $\frac{\mathrm{d} E}{\mathrm{dt}}=25 \mathrm{kV} / \mathrm{cm} \cdot \mathrm{s}$. The resulting total current density $j_{\text {tot }}$ consists of the addition of the displacement current $j_{\text {dis }}$ and the leakage current $j_{\text {leak }}$ according to

$$
j_{\text {tot }}=j_{\text {dis }}+j_{\text {leak }}=\varepsilon_{r} \varepsilon_{0} \frac{\mathrm{d} E}{\mathrm{dt}}+j_{\text {leak }} .
$$

In this equation $\varepsilon_{r}$ and $\varepsilon_{0}$ are the dielectric constants for the material and the vacuum respectively. In the resulting graph the constant part of the curve is due to $j_{\text {dis }}$ which depends on the dielectric material constant and the electrical field strength ramp. The slope results from $j_{\text {leak }}$. The breakthrough field strength is defined as the value when the current density reaches $j_{\text {tot }}=10^{-6} \mathrm{~A} / \mathrm{cm}^{2}$.

The measured current densities over the electrical field strength of the different materials are presented in figure 2. Since the maximum voltage $U_{\max }$ of the used picoamperemeter is limited to $U_{\max }= \pm 100 \mathrm{~V}$, the maximum applied electrical field strength $E_{\max }=U_{\max } / d$ for each capacitor depends on the dielectric thickness $d$. From figure 2 it can be seen that the current density for the spin coated polyacyryl and polyimide increases strongly with the applied electrical field. For $\mathrm{SiO}_{2}$ and $\mathrm{Si}_{3} \mathrm{~N}_{4} j_{\text {tot }}$ is influenced only by $j_{\text {dis }}$ for a wide area before the breakthrough occures. Both anodically oxidized dielectrics $\mathrm{Al}_{2} \mathrm{O}_{3}$ and $\mathrm{Ta}_{2} \mathrm{O}_{5}$ show an unsymmetry between positive and negative field strength. While a definite breakthrough can be observed in the negative region it is not well defined for positive field strength. The definite breakthrough in the negative region can be related to the direction of the applied electrical field strength, which is the same as during the oxidation process. We assume that negative anions in the oxide are causing the undefined breakthrough for positive field strengths.

Table 1 summarizes the thicknesses, the dielectric constants $\varepsilon_{r}$, the breakthrough field strengths $E_{\mathrm{d}}$ and the average roughness $R_{\mathrm{a}}$ determined by atomic force microscope (AFM) measurements for the investigated materials. The average roughness of anodically oxidized dielectrics is higher than for the spin coated and for $\mathrm{SiO}_{2}$ layers but it is comparable to the roughness values measured on $\mathrm{Si}_{3} \mathrm{~N}_{4}$. The anodically oxidized materials have been further investigated due to their electrical performance, the low deposition temperature, the high dielectric constants and the possibility to form thin layers which reduces the required OTFT operating voltages.

Table 1. Thickness, dielectric constant, breakthrough field strength and surface roughness of investigated dielectrics.

\begin{tabular}{rccccc}
\hline material & thickness $[\mathrm{nm}]$ & $\varepsilon_{r}$ & $E_{\mathrm{d}}^{+}[\mathrm{MV} / \mathrm{cm}]$ & $E_{\mathrm{d}}^{-}[\mathrm{MV} / \mathrm{cm}]$ & roughness $R_{a}[\mathrm{pm}]$ \\
\hline $\mathrm{Al}_{2} \mathrm{O}_{3}$ & 60 & 9 & $\approx 5$ & 6 & 935 \\
$\mathrm{Ta}_{2} \mathrm{O}_{5}$ & 50 & 27 & 2 & 4.5 & 1150 \\
$\mathrm{SiO}_{2}$ & 100 & 3.9 & 8 & $>10$ & 667 \\
$\mathrm{Si}_{3} \mathrm{~N}_{4}$ & 200 & 7.5 & $>7$ & $>7$ & 978 \\
polyimide & 400 & 3.9 & $<3$ & 2 & 561 \\
polyacryl & 400 & 3.9 & $<4$ & 3 & 521 \\
\hline
\end{tabular}




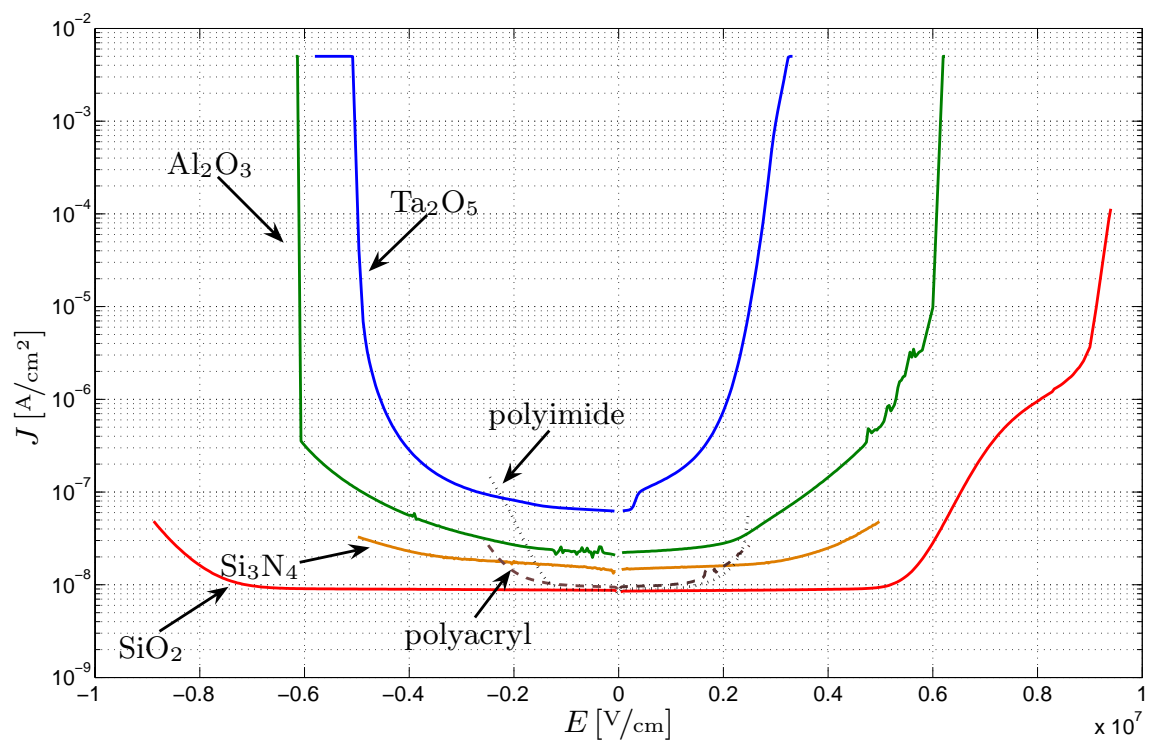

Figure 2. Measured total current density $j_{\text {tot }}$ depending on the electrical field strength of different dielectric materials.

To improve the electrical and structural properties of anodically oxidized dielectrics, different electrolytes are investigated. The results for the electrical performance are summarized in table 2 and in figure 4(a). Characteristic anions which are included in the ethyleneglycol and aqueous citric acid electrolytes are supposed to be incorporated in the dielectric during oxidation and negatively influence the breakthrough performance. ${ }^{8}$ By using $\mathrm{H}_{2} \mathrm{O}_{2}$ as electrolyte the electrical performance of both $\mathrm{Al}_{2} \mathrm{O}_{3}$ and $\mathrm{Ta}_{2} \mathrm{O}_{5}$ is improved especially for positive field strengths. Beside that the average roughness $R_{\mathrm{a}}$ is decreased with the use of $\mathrm{H}_{2} \mathrm{O}_{2}$.

Table 2. Thickness and breakthrough field strength dependenting on the electrolyte.

\begin{tabular}{rccccc}
\hline material & electrolyte & thickness $[\mathrm{nm}]$ & $E_{d}^{+}[\mathrm{MV} / \mathrm{cm}]$ & $E_{d}^{-}[\mathrm{MV} / \mathrm{cm}]$ & $R_{a}[\mathrm{pm}]$ \\
\hline $\mathrm{Al}_{2} \mathrm{O}_{3}$ & ethylenglycol & 60 & $\approx 3$ & 5.5 & 1570 \\
$\mathrm{Al}_{2} \mathrm{O}_{3}$ & $\mathrm{H}_{2} \mathrm{O}_{2}$ & 60 & $\approx 5$ & 6 & 935 \\
$\mathrm{Ta}_{2} \mathrm{O}_{5}$ & citric acid & 100 & 0.1 & 0.5 & 1150 \\
$\mathrm{Ta}_{2} \mathrm{O}_{5}$ & $\mathrm{H}_{2} \mathrm{O}_{2}$ & 50 & 2 & 4.5 & 997 \\
\hline
\end{tabular}

\subsection{Kiessig Interference measurements}

The structure of $\mathrm{Al}_{2} \mathrm{O}_{3}$ is examined by Kiessig Interference measurements. For this measurement the substrate is radiated with a x-ray under small angles of incidence. The x-ray penetrates into the materials and is partly reflected at the layer interfaces. The thickness of the different layers is obtained from the distance between two of the occuring interferences by using equation 4 wherein $d$ is the thickness and $q_{\perp i}$ and $q_{\perp i-1}$ are the orthogonal wave vectors of two proximated interference maxima:

$$
d=\frac{2 \cdot \pi}{q_{\perp i}-q_{\perp i-1}}
$$

The damping of the curve depends on the surface roughness of the investigated layers. 
As shown in figure 3 the structural performance of $\mathrm{Al}_{2} \mathrm{O}_{3}$ is improved with the use of $\mathrm{H}_{2} \mathrm{O}_{2}$. From the reduced damping of the oscillation for the $\mathrm{H}_{2} \mathrm{O}_{2}$ electrolyte it can be concluded that the smoothness of the surface and the transition region between the metal and the oxide are enhanced. The thickness $d$ of the oxide layer can be extracted with equation 4. It is $d=65 \mathrm{~nm}$ for the $\mathrm{Al}_{2} \mathrm{O}_{3}$ grown in the $\mathrm{H}_{2} \mathrm{O}_{2}$ electrolyte and $d=57 \mathrm{~nm}$ when using Ethylenglycol. Both $\mathrm{Al}_{2} \mathrm{O}_{3}$ layers are not stoichimetric as it can be concluded from the maxima and minima positions.

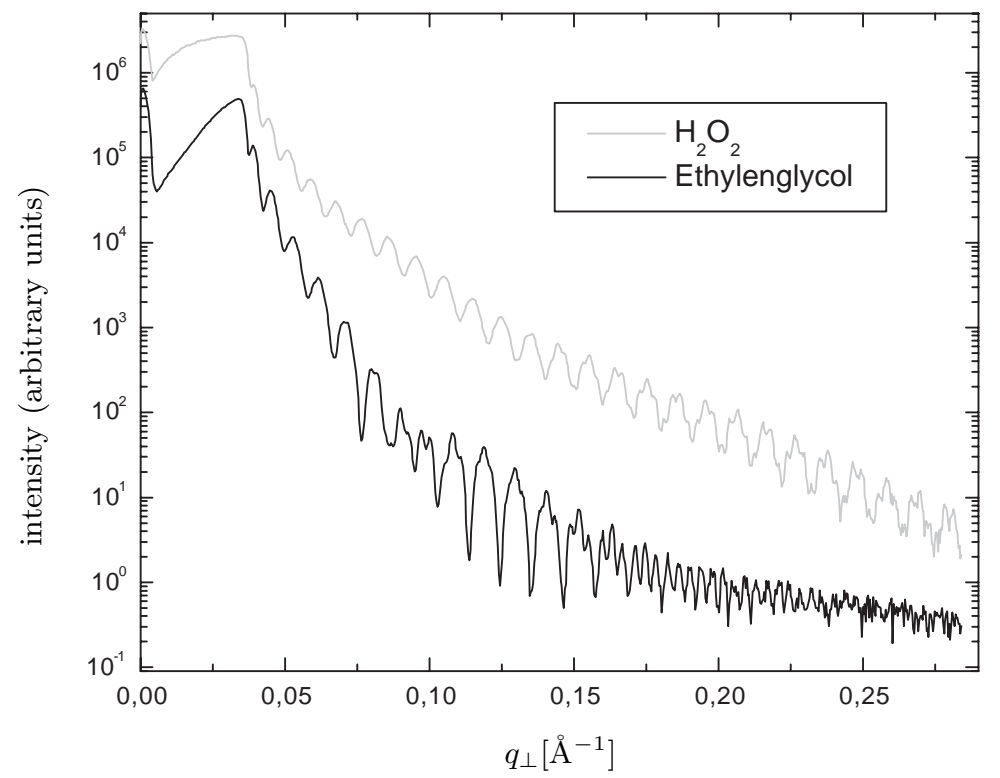

Figure 3. Kiessig oscillation of $\mathrm{Al}_{2} \mathrm{O}_{3}$ samples using different electrolytes.

\subsection{Transfer to plastic substrates}

After optimizing the process for $\mathrm{Al}_{2} \mathrm{O}_{3}$ on glass substrates, it was transferred to a polyethersulphone (PES) plastic substrate. The anodisation of aluminum is choosen as the first process to be transfered on plastic substrate since the bandgap, which influences the intrinsic breakdown, of $\mathrm{Al}_{2} \mathrm{O}_{3}$ is $8.8 \mathrm{eV}$ in comparison to $\mathrm{Ta}_{2} \mathrm{O}_{5}$ where it is $4.4 \mathrm{eV}^{9}$ Additionally, aluminum can be structured by wet-etching while plasma etching processes required for tantalum are affecting the plastic. Thus none of the processes required for fabricating capacitors with $\mathrm{Al}_{2} \mathrm{O}_{3}$ dielectric on plastic (sputtering and structuring the metals, anodisation with electrolytes) has a visible detrimental effect on the plastic substrates. The electrical performance of capacitors with $\mathrm{Al}_{2} \mathrm{O}_{3}$ as insulator are measured on glass and plastic substrates. The results are shown in figure 4(b). The breakthrough behaviour is not influenced by the substrate material. The leakage current determined by the slope of the current density is even less than on the glass substrate. The structure of the anodized films on both substrates are analysed again with Kiessig interference. The resulting characteristics are shown in figure 5.

The resulting thickness extracted from the oscillation caused by the $\mathrm{Al} / \mathrm{Al}_{2} \mathrm{O}_{3}$ interface is $d=63 \mathrm{~nm}$ on the plastic substrate. The thickness is not influenced by the substrate material. From the higher damping of the curve measured on plastic substrate it can be concluded that the surface of the different layers on the plastic substrate are rougher than on glass. The less defined absorption edge measured on the plastic substrate is related to fixture problems resulting in a not perfectly flat substrate during the measurement.

\subsection{AFM measurements}

Since the surface roughness influences the growth of the following layers, it is examined with an atomic force microscope (AFM). Figure 6(a) and 6(b) show the resulting picture of $\mathrm{Al}_{2} \mathrm{O}_{3}$ on glass and on plastic substrates. While small grains are visible on the $\mathrm{Al}_{2} \mathrm{O}_{3}$ surface on plastic the surface on glass is smooth. This indicates 


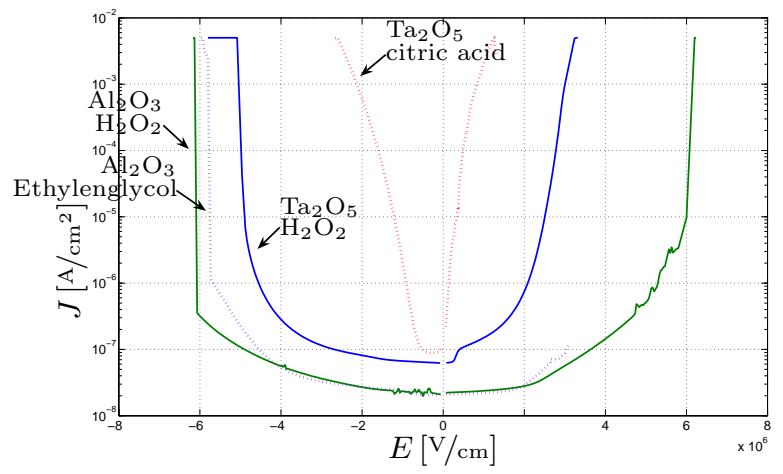

(a) measured for different electrolytes.

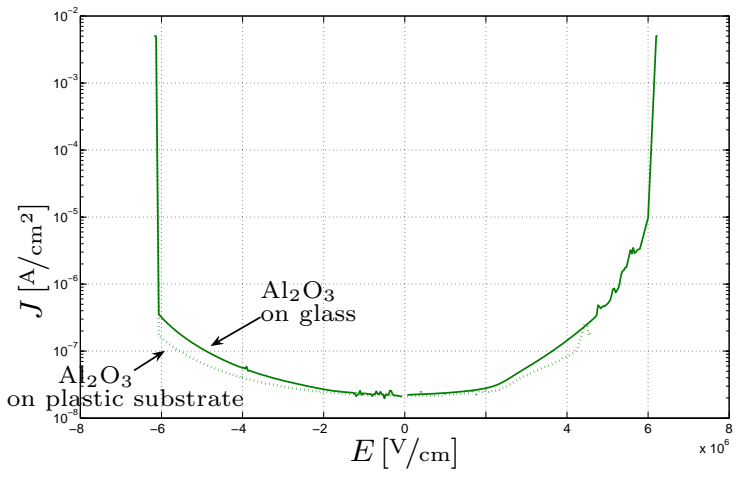

(b) measured on glass and plastic substrates.

Figure 4. Measured total current density depending on the electrical field strength for anodically oxidized dielectrics.

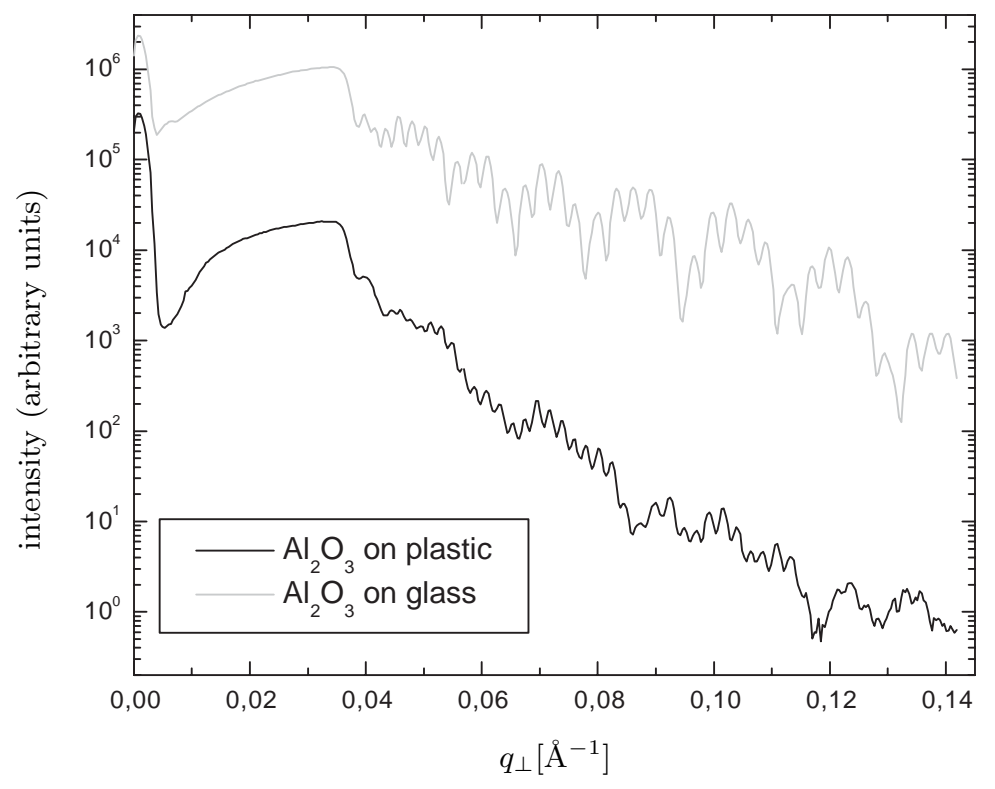

Figure 5. Kiessig oscillations of $\mathrm{Al}_{2} \mathrm{O}_{3}$ measured on glass and plastic substrates.

different growth mechanisms for the sputtered aluminum layer on PES and glass. The resulting values for the average surface roughness $R_{\mathrm{a}}$ of $\mathrm{Al}_{2} \mathrm{O}_{3}$ are $R_{\mathrm{a}}=935 \mathrm{pm}$ on glass and $R_{\mathrm{a}}=1610$ pm on plastic (table 3). The difference in the surface roughness corresponds to the results of the Kiessig interference measurements (figure 5).

\subsection{OTFT performance}

The $\mathrm{Al}_{2} \mathrm{O}_{3}$ is used as the dielectric layer in organic thin film transistors (OTFT). OTFT structures are fabricated on glass and plastic substrates. Bottom gate, bottom contact OTFTs are processed using aluminum as gate metallization. The surface of the structured aluminum is anodized forming the oxide layer. As drain- and source contact material gold is sputtered and patterened. Finally, pentacene is evaporated as semiconducting layer. The cross section of the fabricated OTFTs is presented in figure 7 . For the OTFTs a channel length of $l=5 \mu \mathrm{m}$ and $l=10 \mu \mathrm{m}$ is choosen and the channel width is varied between $w=50 \mu \mathrm{m}$ and $w=850 \mu \mathrm{m}$. The mobility $\mu$ 


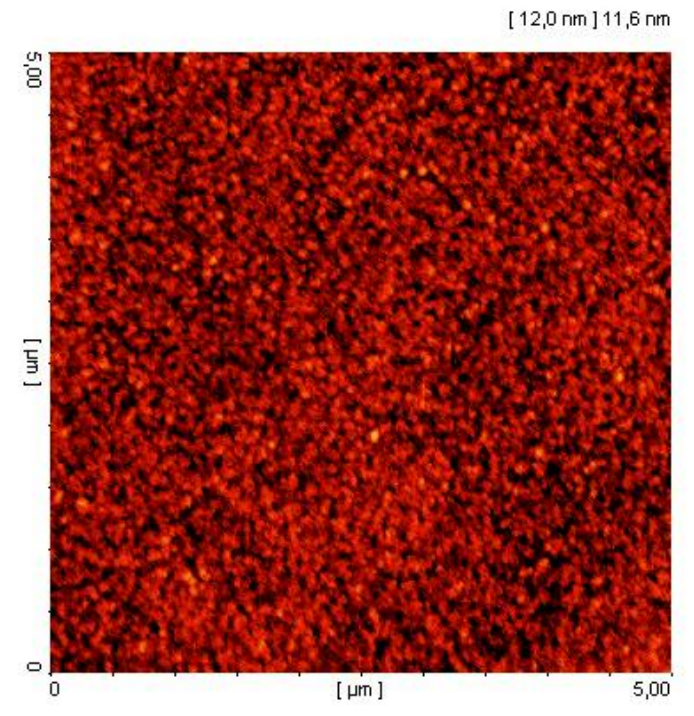

(a) $\mathrm{Al}_{2} \mathrm{O}_{3}$ on glass substrate

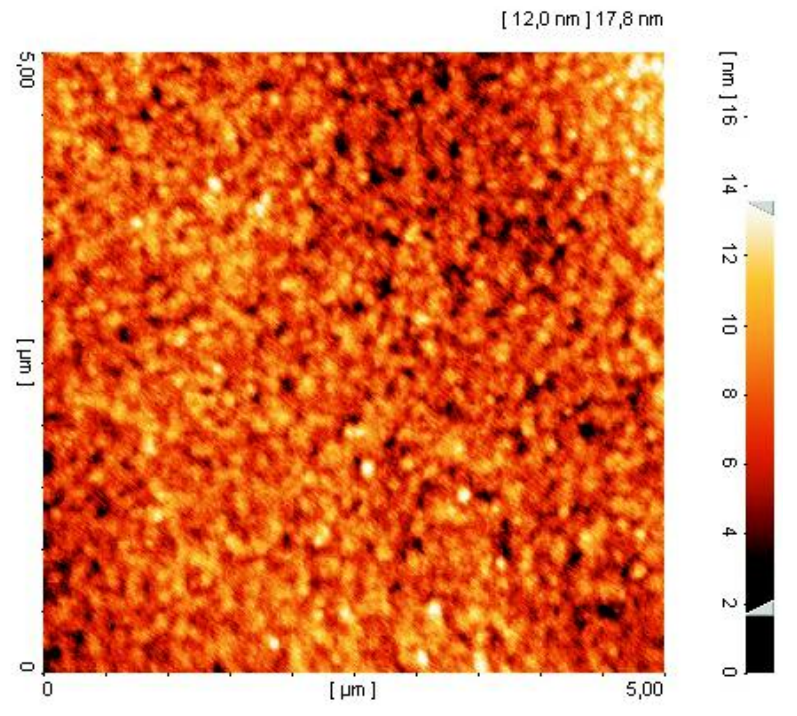

(b) $\mathrm{Al}_{2} \mathrm{O}_{3}$ on plastic substrate

Figure 6. AFM measurements of $5 \mu \mathrm{m} \cdot 5 \mu \mathrm{m} \mathrm{Al} \mathrm{Al}_{2} \mathrm{O}_{3}$ surfaces.

and the threshold voltage $V_{\mathrm{th}}$ are extracted from saturation region of the transfer characteristic using equation 1 . The ON/OFF ratio is based on the maximum and the minimum value of $I_{\mathrm{DS}}$. The resulting values of the OTFT on plastic and glass substrates are compared with each other.

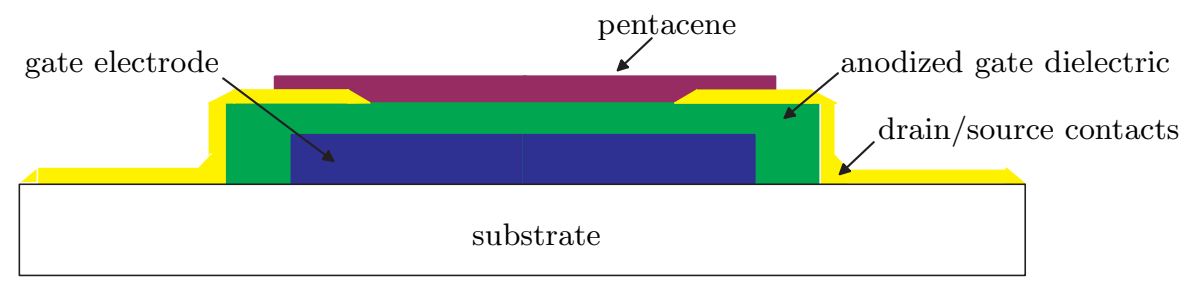

Figure 7. Schematical OTFT set-up.

The distortion which occurs during the process on plastic substrates is reduced by two tempering steps, one before the first layer has been deposited and the other one after the anodisation. The first tempering step is for the preshrinking of the foil to reduce film stress between the aluminum layer and the substrate. The second one is to drive out the moisture which gets into the substrate during the anodic oxidation.

The resulting transfer characteristic of OTFT on glass and plastic substrates are presented in figures 8(a) and $8(\mathrm{~b})$. The extracted parameters for the mobility $\mu$, the threshold voltage $V_{\text {th }}$ and the ON/OFF ratio of the presented characteristics are shown in table 3 .

The electrical performance of these OTFTs on glass and plastic substrates are comparable. Remarkable is the excellent blocking behaviour resulting in low off-currents $I_{\mathrm{OFF}}<1 \cdot 10^{-12} \mathrm{~A}$ for both substrates. $I_{\mathrm{OFF}}$ is not inflenced by leakage currents through the optimized gate dielectric $\mathrm{Al}_{2} \mathrm{O}_{3}$. The mobility and the ON/OFF ratio are not influenced by the substrate material as it can be seen from the values in table 3 . The values are uniformly distributed over the 2 inch test substrates and they are reproducible. Another interesting effect is the threshold voltage shift when measuring from positive to negative gate voltages followed by a reverse sweep. This effect occurs for both substrate materials but is more distinct on plastic. This shift depends on the value of the most negative gate voltage which is applied to the OTFT. Reasons for this shift could be the interface defects 


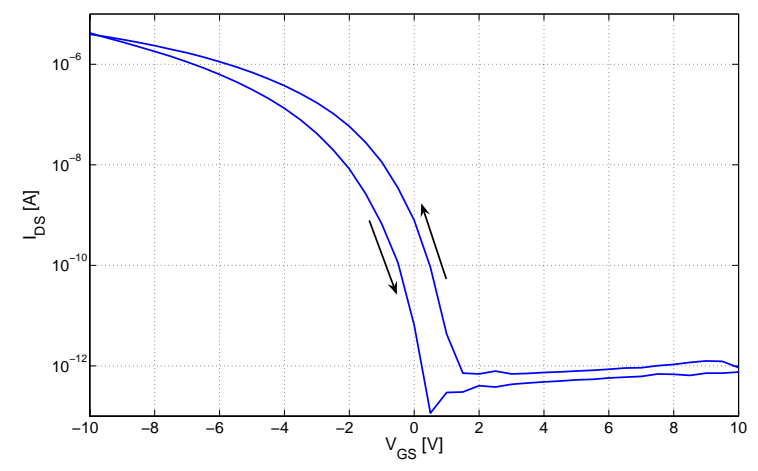

(a) glass substrate

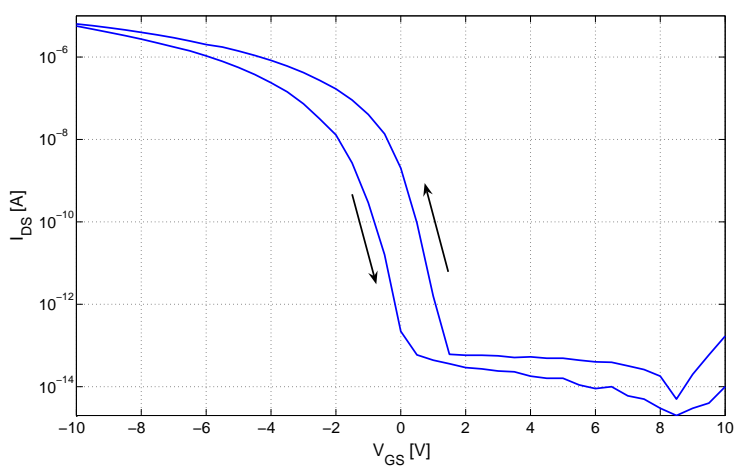

(b) plastic substrate

Figure 8. Transfer Characteristics of OTFTs on plastic and glass substrates. $w=200 \mu \mathrm{m}, l=10 \mu \mathrm{m}, V_{\mathrm{DS}}=-10 \mathrm{~V}$.

Table 3. Extracted values for the mobility $\mu$, threshold voltages $V_{\mathrm{th}}^{-}$and $V_{\mathrm{th}}^{+}$and ON/OFF ratio.

\begin{tabular}{rccccc}
\hline substrate & $\mu\left[\mathrm{cm}^{2} / \mathrm{vs}\right]$ & $V_{\text {th }}^{-}[\mathrm{V}]$ & $V_{\text {th }}^{+}[\mathrm{V}]$ & ON/OFF ratio & $\mathrm{R}_{a}[\mathrm{pm}]$ \\
\hline glass & 0.1 & -2.9 & -1 & $6 \cdot 10^{7}$ & 935 \\
PES & 0.1 & -2.4 & -0.3 & $1 \cdot 10^{8}$ & 1610 \\
\hline
\end{tabular}

between the dielectric and the pentacene. Since the surface of $\mathrm{Al}_{2} \mathrm{O}_{3}$ on plastic substrate is rougher than on glass this would explain the more pronounced shift for OTFTs on plastic substrates. Similar effects in OTFT and different explanations for this can be found in literature. ${ }^{10,11}$ These effects have to be investigated in more detail for a better understanding and further improvements of OTFTs.

\section{CONCLUSIONS}

Different low temperature gate dielectrics are investigated and compared to conventionally used TFT gate dielectrics $\mathrm{SiO}_{2}$ and $\mathrm{Si}_{3} \mathrm{~N}_{4}$ to find a convenient gate dielectric for OTFT application on plastic substrates. Anodically oxidized $\mathrm{Al}_{2} \mathrm{O}_{3}$ is found to fullfill all requirements. Before the process is transferred to plastic substrates the influence of different electrolytes on the electrical and structural properties of the dielectric is investigated. Using breakthrough and x-ray measurements it has been shown that buffered $\mathrm{H}_{2} \mathrm{O}_{2}$ improves both properties. After the process is successfully transferred to plastic substrates, OTFTs on PES are built which show similar electrical behaviour as those on glass substrates.

\section{ACKNOWLEDGMENTS}

This project has been partially funded by the EU funded programme FlexiDis (Contract IST-2004-4354) and the DFG focus programm (project No. PF385/2-3).

The authors thank Stefan Hirschmann from the $3^{\text {rd }}$ Institute of Physics, University of Stuttgart for the purification of pentacene.

\section{REFERENCES}

1. E. Lueder, Liquid Crystal Displays, John Wiley \& Sons, LTD, 2001.

2. H. Klauk, M. Halik, U. Zschieschang, G. Schmid, C. Dehm, R. Brederlow, S. Briole, M. Schütz, S. Maisch, and F. Effenberger, "Molecular thin film transistors with a substhreshold swing of $100 \mathrm{mv} /$ decade," pp. 8.2.14, IEEE International Electron Devices Meeting, 2003. 
3. Y. Inoue, Y. Fujisaki, Y. Iino, H. Kikuchi, S. Tokito, and F. Sato, "Low-Voltage Organic Thin Film Transistors on Flexible Plastic Substrates with Anodized $\mathrm{Ta}_{2} \mathrm{O}_{5}$ Gate Insulators," in Mat. Res. Soc. Symp. Proc. Vol. 736, pp. D4.2.1-6, Material Research Society, 2003.

4. M. Raja, N. Sedghi, S. Higgins, and W. Eccleston, "The benefits of high-k dielectrics for polymer tfts," in Mat. Res. Soc. Symp. Proc. Vol. 734, pp. B9.1.1-6, Material Research Society, 2003.

5. D. Knipp, R. Street, B. Krusor, J. Ho, and R. Apte, "Influence of the dielectric on the growth and performance of pentacene thin film transitors," Mat. Res. Soc. Symp. Proc. 708, pp. 251-256, 2002.

6. H. Baur, S. Jelting, N. Benson, and N. Fruehauf, "An ltps active-matrix process without ion doping," in SID'05, Society for Information Display, 2005.

7. Berry, Hall, and Harris, Thin Film Technology, D. van Nostrand Company, inc, 1968.

8. K. Ozawa and T. Majima, "Anodization behavior of al, and physical and electrical characterzation of its oxide films," Journal of Applied Physics 80, pp. 5828-5836, November 1996.

9. H.-S.-P. Wong, "Beyond the conventional transistor," IBM J. RES. \& DEV. 46(2,3), pp. 133-168, 2002.

10. R. Street, A. Salleo, and M. Chabinyc, "Bipolaron mechanism for bias-stress effects in polymer transistors," Physical Review B 68, p. 085316, 2003.

11. H. Torres, D. Taylor, and E. Itoh, "Interface states and depletion-induced threshold voltage instability on organic metal-insulator-semiconductor structures," Applied Physics Letters 85(2), pp. 314-316, 2004. 